\title{
Third-order Single-bit Sigma Delta modulator structure for an RF reception chain for a LTE network
}

\author{
${ }^{1}$ Soumaya Azzouni, ${ }^{2}$ Nizar Khitouni, ${ }^{3}$ Med Salim Bouhlel \\ ${ }^{1}$ PhD student, Sciences of Electronics Technologies of Information and Telecommunications, higher institute of biotechnology of sfax, Route de Soukra km 4, BP \\ 2613000 , Tunisia. \\ 2Professor, Sciences of Electronics Technologies of Information and Telecommunications, higher institute of biotechnology of sfax, Route de Soukra km 4, BP 261 \\ 3000, Tunisia. \\ ${ }^{3}$ Professor, Sciences of Electronics Technologies of Information and Telecommunications, higher institute of biotechnology of sfax, Route de Soukra km 4, BP 261 \\ 3000, Tunisia.
}

Correspondence Author: Nizar Khitouni, University of Sfax, Sciences of Electronics Technologies of Information and Telecommunications, higher institute of biotechnology of sfax Route de Sokra km 4 BP. 2613000 Tunisia

E-mail: nizar.khitouni@enis.rnu.tn

Received date: 15 April 2018, Accepted date: 15 June 2018, Online date: 15 July 2018

Copyright: $\odot 2018$ Nizar Khitouni et al. This is an open-access article distributed under the terms of the Creative Commons Attribution License, which permits unrestricted use, distribution, and reproduction in any medium, provided the original author and source are credited.

Abstract
The sigma delta conversion technique has been in existence for many years, but recent technological advances now make the
devices practical and their use is becoming widespread. The converters have found homes in such applications as
communications systems, Long Term Evolution (LTE), consumer and professional audio, industrial weight scales, and
precision measurement devices. This paper presents a third-order Sigma Delta modulator for a LTE receiver. LTE is the latest
Mobile Telecommunications technology being currently in development and testing phase. Sigma-Delta ADCs are currently
the right choice for high bandwidth systems and require high resolution. The Continuous-time (CT) structure becomes more
advantageous compared to the disceret -time (DT) due to important properties such as implicit anti-aliasing, resistive input
impedance and low power operation. Simulation results reveal that peak signal to noise distortion is 69dB in Matlab
simulation for 10MHz band width.

Key words: Analog-digital, Sigma-delta modulation, continuos, RF, LTE.

\section{INTRODUCTION}

The digital processing performance of signals and communication systems is generally limited by the quality of the input signal (resolution) (Gabriel ANTONESEI et al., 2010). The increasing use in telecommunication digital technique, Long Term Evolution (LTE), like audio applications, supported the analog to digital converter use. With increasing development of LTE (Long Term Evolution) communication application and signal acquisitions, there is a great demand to request signal bandwidths of some megahertz for analog-to-digital converters (ADCs) in the wireless communication.

Forthcoming communication standards like LTE-A specify transmission scenarios using signals with up to $100 \mathrm{MHz}$ bandwidth (Kelly Tchambake et al., 2017) However, it proved that the traditional converters do not reach high performances on a small surface.

Current research in the field of telecommunication progress exceeds the current technological limits and improves the concept of the future system performances considerably: better integration rate, low power, personal communication apparatus size reduced and the capacity of multiplying the communication number. For the signal transmission, we use a conversion technique called modulation such as the coded pulse modulation or the Sigma-delta modulation. The Sigma- delta $(\Sigma \Delta)$ conversion is currently an increasingly widespread technique in the field of Analog/digital conversion. The analog to digital sigma-delta modulation presents a compromise between the high resolution, (more than 12 bits) and the high integration. Although the $\Sigma \Delta$ modulator was introduced in (Xu Y et al., 2010) (R. Suszyński et al., 2013), it did not earn importance until a recent development of the VLSI technology which allows the implementation on much reduced surfaces. Moreover, these converters are simple, robust and well adapted to the high-speed applications. The converters guarantee a high resolution and can be easily implemented on a chip: it is a concept key to improve the communication system performance and to allow the third generation air interface realization. To improve the resolution and accuracy, the converter (A/D) is built of two different structures to convert N most significant bits (MSBs) and M least significant bits (LSBs), respectively (K. Wawryn et al 2013). This paper is organized as follow. Section II introduces the receiver architecture, including the description of LTE Network. In Section III, the modulator topology and the architecture proposed are presented. Finally, Simulation results and conclusions are given in Section IV and Section V, respectively.

\section{Reception Chain:}

The Radio Frequency receiver chain is generally constituted by: an antenna, a low noise amplifier, mixer, filters and analog-to-digital converter. An ADC is an electronic structure that transforms the DC signal into a series of discrete numerical values. The main characteristics of the ADC are resolution, sampling frequency, linearity and consumption. The receiver lowers the RF frequency to a lower intermediate frequency to facilitate information retrieval. Generally, there are three main families of receiver architecture. Depending on the intermediate frequency value: Heterodyne Receiver, Homodyne Receiver, and Low Intermediate Receptor. The heterodyne architecture is the oldest and used for most cell phones in the past. It is called Superheterodyne when transposing frequency into several steps. The principle of this architecture is presented in the figure, it begins by filtering the RF signal received by a band selection filter and then amplifying it by a low noise amplifier (LNA), then filtered by a rejection filter images. The IR filter output is converted to the intermediate frequency (IF) via a mixer. Then a channel filtering is performed. A homodyne receiver is also referred to as direct conversion or Zero-IF. This architecture has a simpler structure than that of the heterodyne receptor. The principle consists of transposing the baseband signal directly using an IQ block. The resulting baseband signal is then filtered before passing through the ADC. 


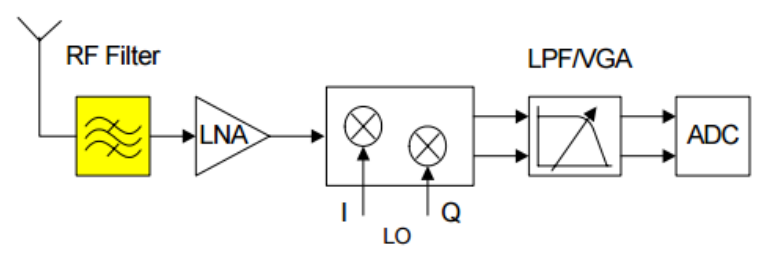

Fig. 1: Architecture of a homodyne receiver.

The low-frequency intermediate receiver architecture is based on the same principle of the homodyne receiver, the transposition of RF signal in the baseband, to a non-zero but fairly low intermediate frequency. This reintroduces the problem of image frequency that can removed by an image suppression technique. Each receiver architecture has major advantages and disadvantages. The simplest architecture is aimed at with low cost and low energy consumption. The heterodyne receiver offers high selectivity and sensitivity but suffers from the image frequency problem. The use of the various filters to respond to this problem increases the complexity of realizing a system especially at high frequencies. The high number of components increases energy consumption and makes it difficult to adapt to the system requirements of a single integrated chip required by communication systems today. The same image frequency problem occurs in the low-frequency intermediate architecture. The choice of the homodyne architecture is justified by the simplicity, absence of image frequency problem that the addition of filters and convenience with systems on chips require. However, this architecture has disadvantages that can be alleviated by the implementation of different methods (MAK et al., 2007).

\section{LTE Network:}

LTE (Long Term Evolution) is the name of a project within the third Generation Partnership Project (3GPP) under release 8 name to produce the fourth generation (4G) mobile network standard technical specifications. In fact, it is a system that aims to improve the performance of third-generation networks, and it increases service delivery and diversification by bringing about significant changes. This evolution brings many technical advantages for cellular networks. The bandwidth is scalable, ranging from $1.25 \mathrm{MHz}$ to $20 \mathrm{MHz}$, in order to adapt to the needs of operators of distinct networks with different bandwidth allocations. Release 9 is based on the development of the missing features found in previous releases, while release 10 focuses on the development of "LTE Advanced" (the real 4G) that is included in the family IMT-advanced (International Mobile Telecommunications-Advanced) introduced by the ITU (International Telecommunication Union). The LTE radio interface has introduced a number of new technologies, enabling the efficient use of spectrum and providing much higher data rates. For example, the LTE used Orthogonal Frequency Division Multiplex (OFDM) technology as a signal carrier and the associated access regimes, OFDMA (Single Frequency Division Multiple Access), SC-FDMA (Single Frequency Division Multiple Access) and MIMO (Multiple Input Multiple Output) (DAHLMAN et al., 2013).

With OFDM technology, LTE allows dynamic assignment of number of subcarriers for a user according to the traffic load. With the advantage of flexibility in the use of the frequency band (different sizes including $1.25,2.5,5,10,15$ and $20 \mathrm{MHz}$ ).

Compared with traditional antenna techniques that improve channel quality, MIMO is an advanced antenna technology that allows multiple parallel transmissions (orthogonal channels) through the use of multiple antennas at the receiver and the transmitter. The increase in quality is proportional to the number of antennas. MIMO is also a spatial diversity technique that increases system capacity and user throughput without transmission energy and no additional bandwidth.

Table 1: LTE Features.

\begin{tabular}{|c|c|c|c|c|c|c|}
\hline Bandwidth(MHz) & 1.4 & 3 & 5 & 10 & 15 & 20 \\
\hline Sub-carrier spacing (Khz) & \multicolumn{6}{|l|}{15} \\
\hline Sampling frequency (MHz) & 245.76 & 245.76 & 245.76 & 491.52 & 460.80 & 491.52 \\
\hline Modulation Scheme & \multicolumn{6}{|c|}{ QPSK, 16-QAM, 64-QAM } \\
\hline
\end{tabular}

3. Sigma Delta:

3.1. Overview:

The principle of the Sigma-Delta modulator is based on the concept of oversampling and quantization noise shaping, which is introduced by Culter in 1954. However, the first SDM was invented by Inose et al in 1962. The technique of oversampling involves obtaining a sampling frequency higher than the Nyquist frequency $(2 *$ signal bandwidth). This technique requires a large number of samples in order to reduce the quantification error in the signal band.

$O S R=\frac{F_{S}}{2 F_{b}}$

With Fs and $\mathrm{Fb}$ are the sampling rate and the signal bandwidth respectively.

Quantification noise shaping, Quantification is the process used to associate an analog signal sampled at a discrete level equivalent to a finite number of bits. The quantization noise is the ratio between the actual value and the level that has been associated with it. Noise shaping is the process of filtering quantization errors. This method reduces the power of the quantization error in the frequency band of interest and increases the quantization error power outside that band. The transfer function of this filter is called NTF: Noise Transfer Function. Several architectures are studied in the literature; they are classified according to different strategies:

The nature of the signal: according to this category, there are two types of modulators, depending on the choice of loop filter: LP $\Sigma \Delta$ (low pass) and BP $\Sigma \Delta$ (band pass).

The LP $\Sigma \Delta$ modulators use the integrator as a loop filter, but the BP $\Sigma \Delta$ use resonators. Therefore, analysis and synthesis are the same only difference is the nature of filter. It is possible to obtain a BP $\Sigma \Delta$ from a LP $\Sigma \Delta$ by applying the transformation method LP to BP.

BP $\Sigma \Delta$ can be a good application for wireless communications systems due to low power consumption. The number of internal quantizer bits: This results in two classes of the modulators: single-bit and multi-bit. The multibit architecture offers a better resolution compared to that of single bit however it suffers from non-linearity. Different techniques are proposed to solve this problem, the most well known of which is the DEM: Dynamic Element Matching. Another solution is the Dual-quantization architecture which is a combination between the two topologies in order to complete the advantages of both. The number of quantifiers: this category results in Single-loop architecture with only a single quantifier, Cascade architecture and Dual Quantization architecture. The Cascade or Multistage architecture is formed by several stages, each stage being constituted by a low-order (first or second) $\Sigma \Delta$ single-loop modulator modulated by the signal containing the quantification error of the preceding stage. The loop filter circuit nature: There are two classes of CT (Continuous Time) and DT (Discrete Time). The DT $\Sigma \Delta$ where the loop filter samples the signal at the phase of the modulator input which requires a filter for aliasing. However for CT $\Sigma \Delta$, the Loop filter samples the signal at the phase of the quantizer input (loop filter output). 

and Applied Sciences, 12(7): 14-18.

Although the DT implementation is the most widely used based on SC: Switched-Capacitor circuits, the CT implementation is becoming a good architecture for application in broadband communication systems offered by a high OSR with low energy consumption. A method of performing a CT $\Sigma \Delta$ from an equivalent DT $\Sigma \Delta$ using a DT-to-CT transformation method (JOSÉ M., 2011).

\subsection{Modulator Performance:}

Signal to Noise Ratio (SNR) is the ratio of the input signal power to the useful band noise power:

$\operatorname{SNR}(d B)=10 \log \frac{P_{\text {Signal }}}{P_{\text {noise }}}$

The Signal to Noise and Distortion Ratio (SNDR) is given by:

$\operatorname{SNDR}(d B)=10 \log \left(\frac{P_{\text {Signal }}}{P_{\text {noise }}+P_{\text {distorsion }}}\right)$

$\mathrm{P}_{\text {signal }}$ is the signal power at the output of the modulator, $\mathrm{P}_{\text {noise }}$ and Pdistortions the power of the harmonics.

The dynamic range of the ADC is a measure of the range of input amplitudes for which the structure always operates correctly (JOSÉ M., 2011).

$D R=6.02+1.736(d B)$

ENOB (Effective Number of Bits) is the number of actual bits measured by an input signal, the value of this metric is given by [4].

$\mathrm{ENOB}=\frac{\mathrm{SNR}(\mathrm{dB}) 1.76}{6.02}$

The ERBW (effective resolution bandwidth) is equal to the frequency that results in a $3 \mathrm{~dB}$ reduction in the SNR value.

To compare the various converters, we use the value of Merit Factor (FOM: Figure Of Merite) that is calculated by:

$F O M=\frac{2^{E N O B} \cdot 2 \cdot E R B M}{P_{m}}$

With Pm is the power consumed by the converter.

\subsection{Modulator architecture:}

For the same topology, we can implement different implementations. These implementations are generally divided into two main classes (JOSÉ M., 2011): feedback (FB) and feed forward (FF). Each family is presented in Figure 2.

(a)

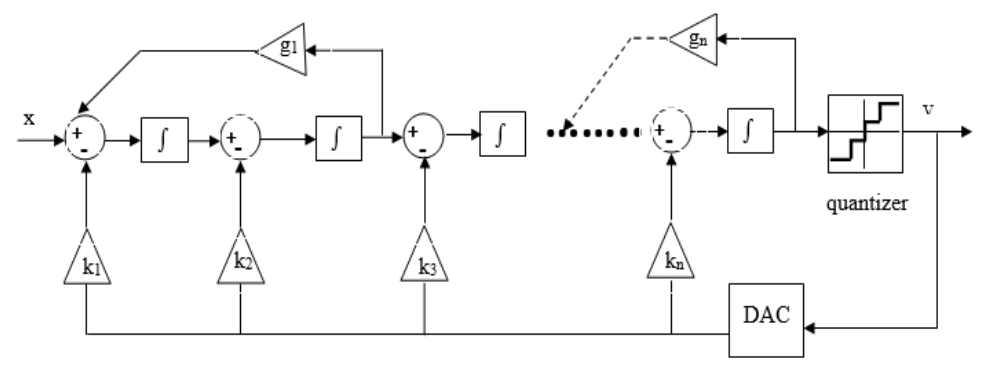

(b)

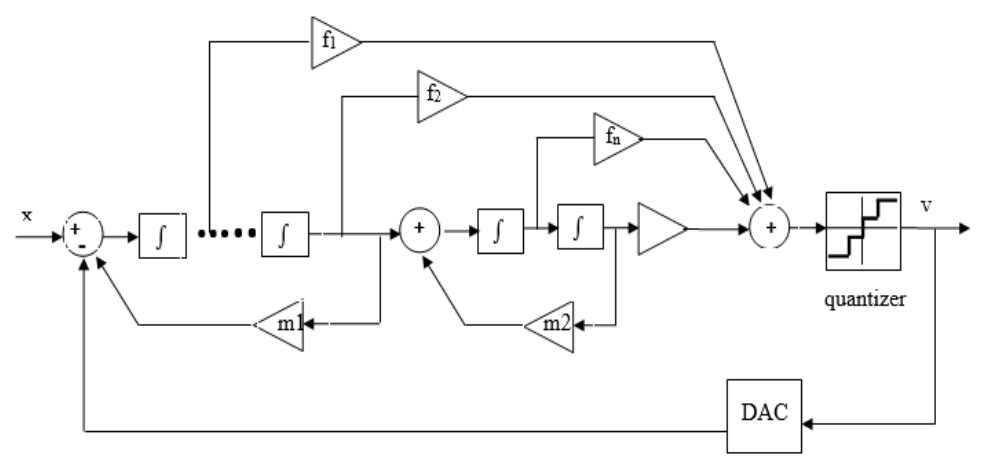

Fig. 2: Architecture (a) Feedback and (b) Feed forward.

The use of internal multibit quantization usually requires linearization techniques in the return DAC. Any error in the level spacing (non-linearity) of a multibit quantizer is directly referenced by the input.

Figure 3 shows the proposed third-order Sigma Delta modulator architecture with single-bit quantizer. The integrators are implanted in the S-domain. On the feedback, NRZ DAC is used with the transfer function (JOSÉ M., 2011).

$H_{D A C}(S)=\frac{1-e^{-S T}}{S}$

The modulator coefficients (a, m1, m2, m3, k1, k2, k3) are calculated by simulation in the aspect of a high SNR. 


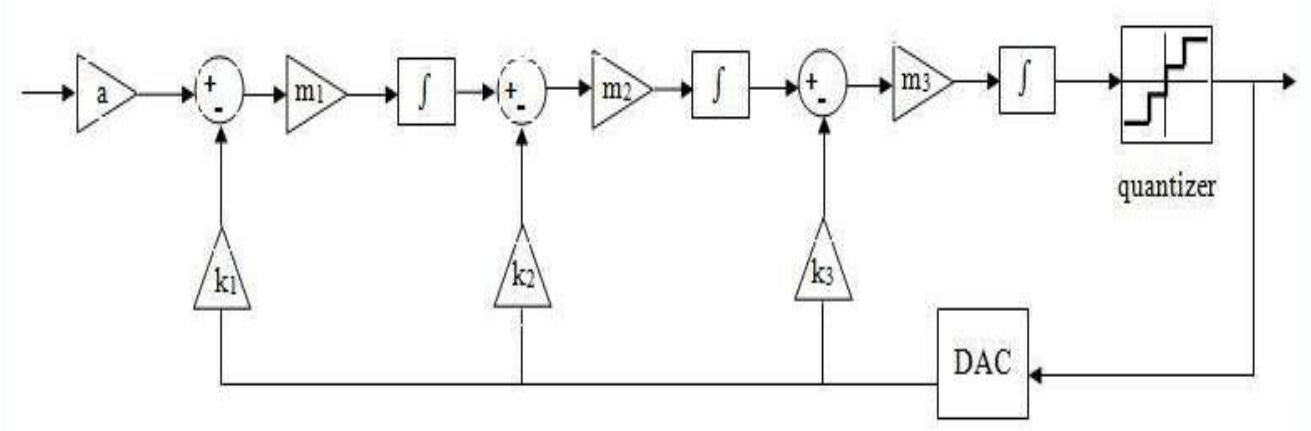

Fig. 3: Proposed modulator architecture.

\section{Simulation results:}

In this section, the simulation results of the proposed architecture are presented using the Simsides (a MATLAB SIMULINK tools). The measured spectrum of the digital output is shown in Fig. 4.

In this simulation, the input signal was $2.1 \mathrm{MHz}$ sine wave, and the sampling frequency was $491.52 \mathrm{MHz}$. The FFT size is 32768 data points and the output data is weighted a Hamming window.

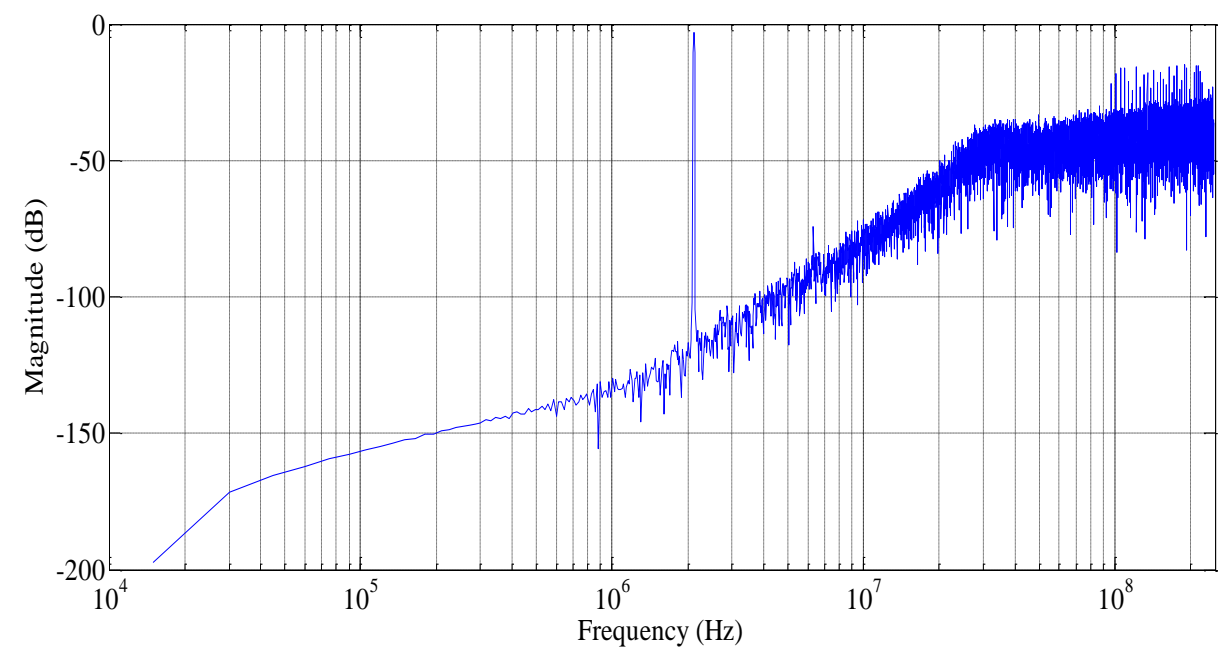

Fig. 4: Measured FFT spectrum.

The continuous-time modulator achieves a DR of $70 \mathrm{~dB}$ and an SNR of $69 \mathrm{~dB}$ within a $10 \mathrm{MHz}$ signal bandwidth. Table 2 summarizes the modulator performance and also lists the comparison with other modulators for wireless networks.

Table 2: Comparison of results.

\begin{tabular}{|c|c|c|c|c|c|c|}
\hline Ref & Fs $(\mathrm{MHz})$ & $\mathrm{BW}(\mathrm{MHz})$ & Order & $\mathrm{SNR}(\mathrm{dB})$ & $\mathrm{DR}(\mathrm{dB})$ & Technology \\
\hline XU Ken 2016 & 491 & 10 & 3 & 62 & 66 & \\
\hline DahlmanErik 2013 & 240 & 5 & 3 & 70 & 71 & CMOS 90n \\
\hline Weng C 2017 & 640 & 20 & 4 & & 58 & CMOS90n \\
\hline Ke Y 20110 & 640 & 10 & 4 & 65 & 67 & CMOS 90n \\
\hline Crombez P 2010 & 1000 & 10 & 3 & 43 & 48 & $0.35 \mathrm{u}$ \\
\hline Hong J 2014 & 288 & 5 & 3 & 71 & 72 & $65 n$ \\
\hline Andersson 2012 & 3000 & & 2 & & & \\
\hline Salgado 2014 & $80 / 160 / 230$ & $2.5 / 5 / 10$ & 3 & $84.8 / 85.8 / 84.5$ & $84.6 / 83.3 / 81.9$ & $65 n$ \\
\hline Xu Y 2012 & 3200 & $45.7 / 53.3$ & 3 & $84.6 / 83.1$ & $90 / 88$ & \\
\hline R.Suszyński 2014 & 640 & 10 & 3 & 65 & 67 & $90 n$ \\
\hline K. Wawryn 2013 & 100 & 1 & 3 & 56.8 & 60 & \\
\hline This work & 491.52 & 10 & 3 & 69 & 70 & CMOS $0.18 \mathrm{u}$ \\
\hline
\end{tabular}




\section{Conclusion:}

In this paper, we have proposed a design modeling and simulation for a continuous-time third-order Single-bit Sigma-Delta modulator for LTE receiver. The continuous time (CT) implementation is becoming a nice architecture for application in broadband communication systems (LTE) since it meets the requirements of the new communication standards in terms of resolution, speed and consumption and offered by a high OSR with low power. The architecture of the modulator use CRFB structure with a NRZ feedback scheme. The simulation of the modulator using the Simsides MATLAB SIMULINK tools. The modulator, clocked at $491.52 \mathrm{MHz}$, achieves a SNR 69dB, which gives a resolution of 11 bits, for $10 \mathrm{MHz}$ band width. The FFT size is 32768 data points and the output data is weighted a Hamming window.

\section{REFERENCES}

Andersson, M., M. Anderson, L. Sundström and P. Andreani, 2012. A $7.5 \mathrm{~mW} 9 \mathrm{MHz}$ CT $\Delta \Sigma$ modulator in $65 \mathrm{~nm}$ CMOS with $69 \mathrm{~dB}$ SNDR and reduced sensitivity to loop delay variations. In Solid State Circuits Conference (A-SSCC), IEEE Asian, pp: 245-248.

Crombez, P., G. Van der Plas, M.S. Steyaert and J. Craninckx, 2010., A single-bit 500 KHz-10 MHz multimode power-performance scalable 83-to-67 dB DR CT $\Delta \Sigma$ for SDR in $90 \mathrm{~nm}$ digital CMOS. IEEE Journal of Solid-State Circuits, 45(6): 1159-1171.

DAHLMAN Erik, PARKVALL Stefan, and SKOLD Johan. 2013 4G: LTE/LTE-advanced for mobile broadband. Academic press.

DAHLMAN Erik, PARKVALL, 2013. 4G: LTE/LTE-advanced for mobile broadband. Academic press.

Gabriel ANTONESEI, Cristina TURCU2, Adrian GRAUR. 2010, Basic Consideration for Signal Processing Solutions Used in Sigma-delta Based ADC and DAC Converters, Advances in Electrical and Computer Engineering, 10(1).

Hong, J.H. and Z.Y. Chen, 2014. A low-power 10MHz bandwidth continuous-time $\Sigma \Delta$ ADC with Gm-C filter. In Circuits and Systems (MWSCAS), IEEE 57th International Midwest Symposium on pp: 853-856. IEEE.

José, M., 2011. Sigma-delta modulators: Tutorial overview, design guide, and state-of-the-art survey. IEEE Transactions on Circuits and Systems I: Regular Papers, 58(1): 1-21.

Ke, Y., P. Gao, J. Craninckx, G. Van der Plas and G. Gielen, 2010. A 2.8-to-8.5 mW GSM/bluetooth/UMTS/DVB-H/WLAN fully reconfigurable CT $\Delta \Sigma$ with 200kHz to 20MHz BW for 4G radios in 90nm digital CMOS. In VLSI Circuits (VLSIC), IEEE Symposium on pp: 153-154.

Kelly Tchambake, Dang-Kièn Germain Pham, Chadi Jabbour, Patricia Desgreys, Patrick Loumeau, 2017. A Multi-Channel $\Sigma \Delta$ Modulator for Subband Digital Predistortion with LTE signals, IEEE International New Circuits and Systems Conference, 1-4.

MAK, Pui-In, U., Seng-Pan and MARTINS, Rui Paulo, 2007. Transceiver architecture selection-review, state-of-the-art survey and case study. AnalogBaseband Architectures And Circuits For Multistandard And Low voltage Wireless Transceivers, pp: 9-40.

Suszyński, R. and K. Wawryn, 2013. Rapid prototyping of algorithmic A/D converters based on FPAA devices”, Bull. Pol. Ac.: Tech., 61(3): 691-696.

SALGADO, Gerardo Molina, DOLECEK, Gordana Jovanovic, MORGADO, Alonso, et al., 2014. "Design considerations of band pass CT $\Sigma \Delta$ modulators for software-defined-radio receivers. In Circuits and Systems (ISCAS), IEEE International Symposium on. IEEE, pp: 718-721.

Wawryn, K. and R. Suszyński, 2013. Low power 9-bit pipelined A/D and 8-bit self-calibrated D/A converters for a DSP system, Bull. Pol. Ac.: Tech., 61(4): 979-988.

Weng, C.H., Y.Y. Lin and T.H. Lin, 2017. A 1-V 5-MHz Bandwidth 68.3-dB SNDR Continuous-Time Delta-Sigma Modulator with a Feedback-Assisted Quantizer. IEEE Transactions on Circuits and Systems I: Regular Papers.

XU Ken, CAI Min, DAGHER, Elias H., 2016. A $10.2 \mathrm{~mW}$ multi-mode continuous-time $\Delta \Sigma$ ADC with $70-87 \mathrm{~dB}$ DR and $0.7-10 \mathrm{MHz}$ bandwidth for TDSCDMA and LTE digital receivers. Analog Integrated Circuits and Signal Processing, 89(2): 395-410.

Xu, Y., B. Chi, Z. Wang, 2012. Power-scalable multi-mode reconfigurable continuous-time lowpass/quadrature bandpass sigma-delta modulator for zero/low-IF receivers. In Circuits and Systems (ISCAS), 2012 IEEE International Symposium on pp: 293-296. IEEE. 